CCASE REPORT

Volume 16 Supp 12021

DOI: 10.21315/aos2021.16.s1.11

ARTICLE INFO

Submitted: 02/03/2021

Accepted: 27/05/2021

Online: 22/09/2021

\section{One-piece Implant Placement as an Alternative and Acceptable Treatment for Missing Tooth Rehabilitation: A Case Report}

\author{
Paulus Budi Teguh", Chaterina Diyah Nanik \\ Department of Prosthodontics, Faculty of Dentistry, Universitas Hang \\ Tuah Surabaya, Kota Surabaya, Jawa Timur 60111, Indonesia
}

${ }^{*}$ Corresponding author: paulte16@gmail.com

To cite this article: Teguh PB, Nanik CD (2021). One-piece implant placement as an alternative and acceptable treatment for missing tooth rehabilitation: A case report. Arch Orofac Sci, 16(Supp.1): 67-72. https://doi.org/10.21315/aos2021.16.s1.11

To link to this article: https://doi.org/10.21315/aos2021.16.s1.11

\begin{abstract}
Currently dental implant treatment become popular. More clinicians are being presented with the opportunity to perform implant services in their own practice. The use of one-piece implant can be considered as treatment for the replacement of missing tooth in the areas of limited tooth-to-tooth spacing and would bring less trauma for the soft tissue. A 52-year-old female patient presented with difficulty in mastication due to the loss of teeth of 35 region with limitation mesio distal space. Radiology examination did not reveal any radiolucencies in 35 region. Radiograph revealed sufficient bone height from crestal to the floor of the mandible. A one-piece implant was placed in the 35 region. The present case used an endosteal implant, one stage surgery, and delayed loading. Restoration with porcelain fused to the metal crown had been delivered three months after the one-piece implant placement. The result of this treatment indicated that one-piece implant could be considered as an effective therapy choice for replacement of missing tooth in order to rehabilitate the masticatory function.
\end{abstract}

Keywords: Limited tooth spacing; less trauma; one-piece implant

\section{INTRODUCTION}

Partial denture and fixed bridge had been widely known as the alternatives to replace one to two missing teeth. Fixed bridge was favourable to replace the missing teeth because it rehabilitated functionally close to natural teeth. Nowadays, fixed prostheses using dental implant become one of the treatment plan alternatives (Powers \& Sakaguchi, 2006). The advantages of dental implant are no preparation to the abutment teeth is needed but they bring out results that are satisfying aesthetically. Besides, dental implant provides the highest convenience for patient. Patients tend to satisfy with the final result, both functional and aesthetic aspect. As compared to the other conservative prosthetic restoration, dental implant has the ability to restore the function as well as the aesthetic appearance for cases of missing teeth (Misch, 2007).

\section{CASE REPORT}

A 52-year-old female patient came to the dental clinic with a chief complaint of difficulty in the mastication due to loss of teeth at posterior left mandibular. She had undergone teeth extraction five years ago. The patient health status was in optimum 
condition and did not suffered from any systemic disease. She opted for implantsupported fixed prostheses. Intra oral examination showed loss of teeth 35,37 . Clinical condition of the patient before treatment were examined (Fig. 1). There was limitation of mesio-distal aspect in 35 area and the remaining roots of 15,16 . She had a good oral hygiene. The patient hoped to improve her mastication function.

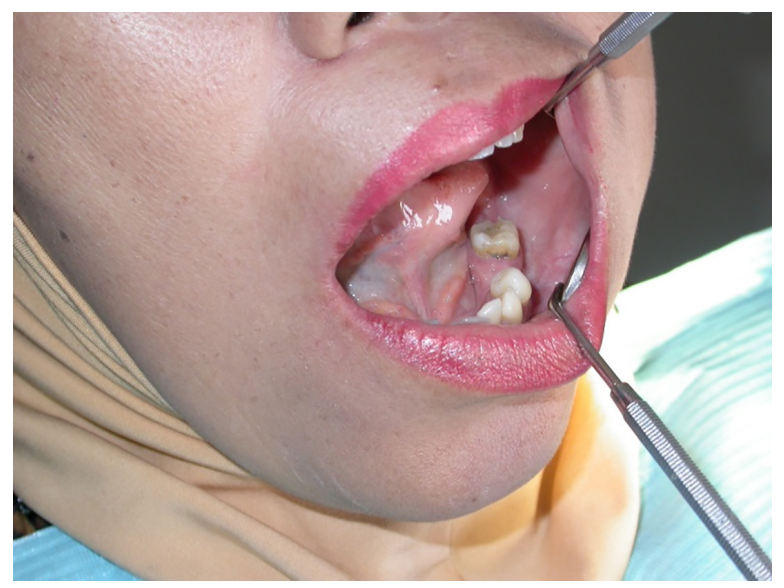

Fig. 1 Clinical condition of the patient before treatment.

Pre-treatment panoramic radiographs (Fig. 2) were taken and revealed that there was no radiolucency in 35 region and sufficient bone height from crestal to the floor of mandible (height of alveolar bone: $40 \mathrm{~mm}$, with estimated bone width: $12 \mathrm{~mm}$ ). There was limitation of mesio-distal aspect (approximately $6 \mathrm{~mm}$ ) of 35 due to mesially tipped of 36. Next, the impression of maxilla and mandible were taken to obtain the diagnostic casts as shown on the Fig. 3. From the evaluation on the diagnostic casts, and the panoramic radiographs, using the bone callipers, the alveolar bone height and width were estimated.

Furthermore, the treatment plan was designed, by choosing the implant abutment and the superstructure for the case. Onepiece implant with $\varnothing 3.0 \mathrm{~mm}$ and $12 \mathrm{~mm}$ length, was planned as the treatment for the present case. Informed consent was obtained from the patient after explanation of the procedure that was to be carried out. The implant placement protocols were as follows: aseptic procedure in the implant site with povidone iodine and followed by mandibular block anaesthesia (2\% lidocaine with 1:80,000 epinephrine). Flapless surgery was then carried out by using a tissue punch in the implant site.

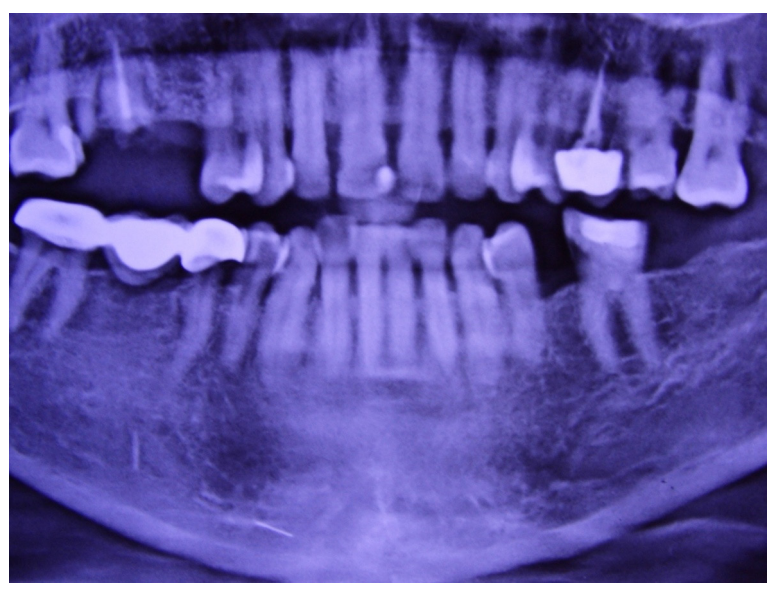

Fig. 2 Panoramic radiograph before treatment.
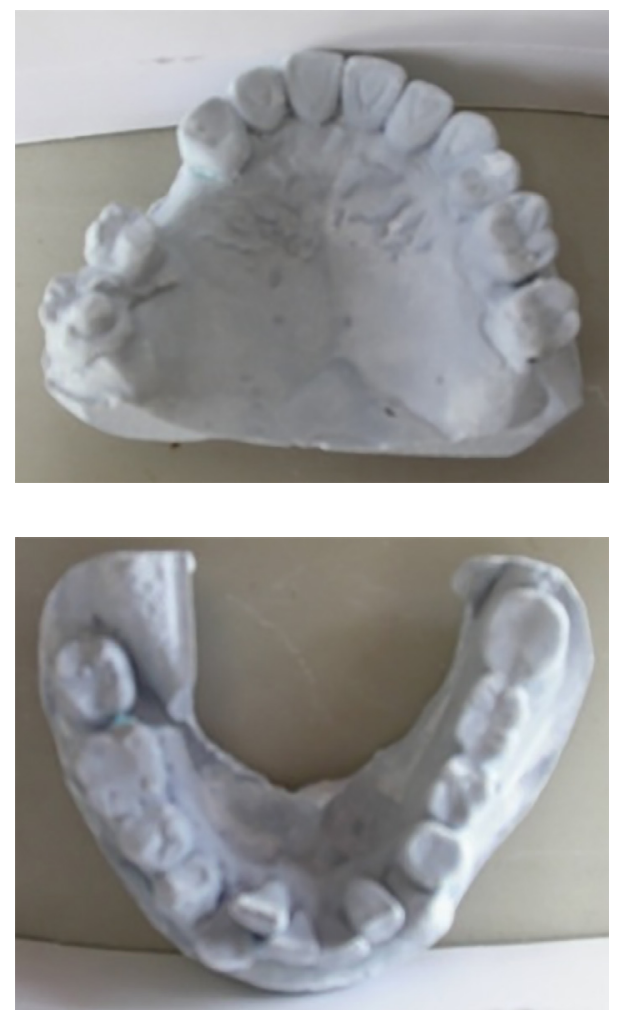

Fig. 3 Diagnostic casts based on the impression of maxilla and mandible. 
The bone was drilled using a lance drill, in $5 \mathrm{~mm}$ depth as a guide to mark out implant site. Bone drilling phase using twist drill (Ø $2.0 \mathrm{~mm}$, depth $12 \mathrm{~mm}$ ) with drill speed of $1,200 \mathrm{rpm}$ to $1,500 \mathrm{rpm}$. After that, the depth of implant site was measured using the depth gauge. Then, a paralleling tool was inserted to check the parallelism with the adjacent teeth. If the parallelism was not appropriate, preparation was carried on using pilot drill (Ø $2.5 \mathrm{~mm}$ ), drill speed of $800 \mathrm{rpm}$ to verify the parallelism of the implant position. After that, preparation with twist drill (Ø $2.5 \mathrm{~mm}$ ), followed by contouring the alveolar bone using countersink (Ø $2.5 \mathrm{~mm}$ ) with drill speed of $750 \mathrm{rpm}$. Later, the surgical tapping site was prepared with bone tap $(\varnothing 2.5 \mathrm{~mm})$. Finally, the insertion of fixture for one-piece implant (Ø $3.0 \mathrm{~mm}, 12 \mathrm{~mm}$ length) with drill speed of $20 \mathrm{rpm}$ and torque $20 \mathrm{Ncm}$ was carried out. Clinical result of implant placement was shown on Fig. 4. Then, panoramic radiograph was carried out to evaluate the fixture implant placement at second premolar left mandibular region as shown on Fig. 5. After the surgery, the patient was prescribed $500 \mathrm{mg}$ of mefenamic acid three times daily for three days and mouthwash three times daily. Patient was told to come on the next day.

\section{First Evaluation Visit}

A day after the implant placement, there was no complaint from the patient. Intra oral examination showed erythematous gingiva around the implant site, with no mobility of the implant. Irrigation by saline was performed and hyaluronic acid $0.2 \%$ gel was given on the gingiva around the implant site. Patient was instructed to maintain her oral hygiene and came for evaluation the next week.

\section{Second Evaluation Visit}

A week after the first evaluation, no complaint was recorded from the patient. Evaluation on the implant body with regard

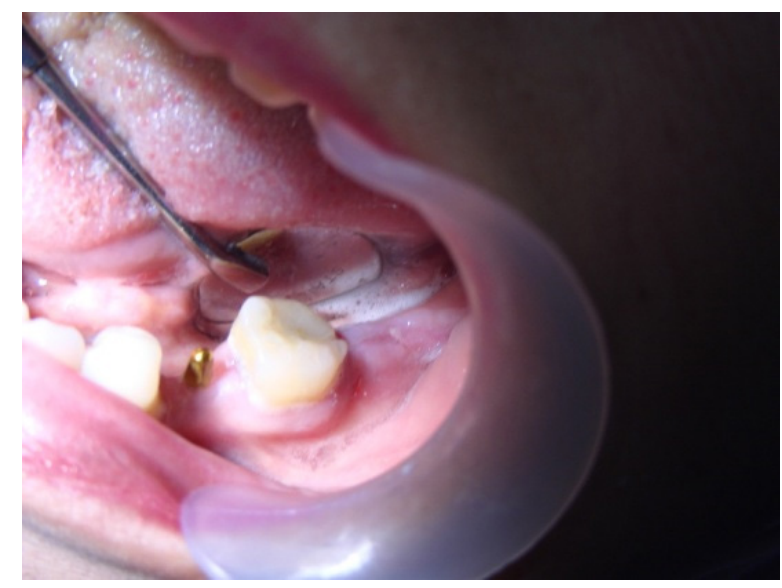

Fig. 4 Clinical condition of the patient after the implant placement.

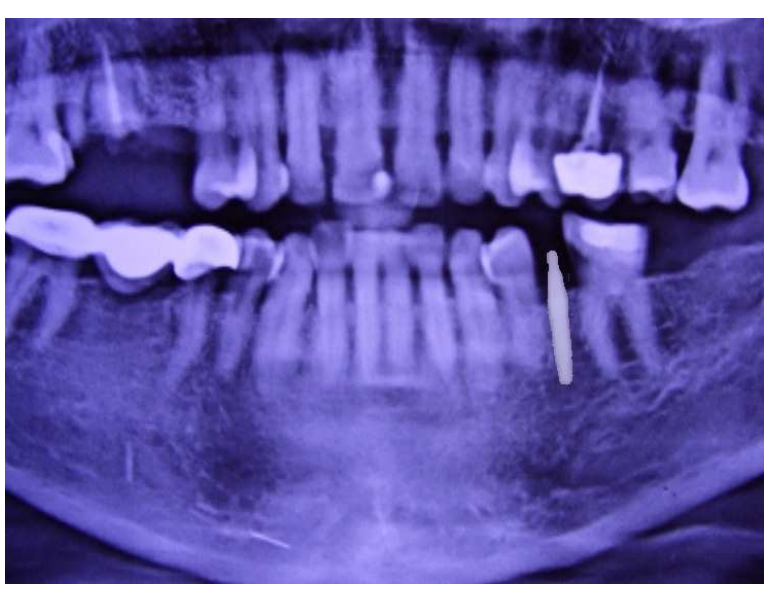

Fig. 5 Panoramic radiograph after implant placement (mandibular left second premolar region).

to percussion test, palpation and mobility had all come out negative. Irrigation with saline was performed and placement of gingival cuff was made to recontour the gingival sulcus area second premolar left mandibular region as shown on Fig. 6 .

\section{Third Evaluation Visit}

Two weeks after the second evaluation, no complaint was recorded from the patient. Evaluation on the implant body with regard to percussion test, palpation, and mobility had all come out negative, and the gingival cuff was in the right place. 


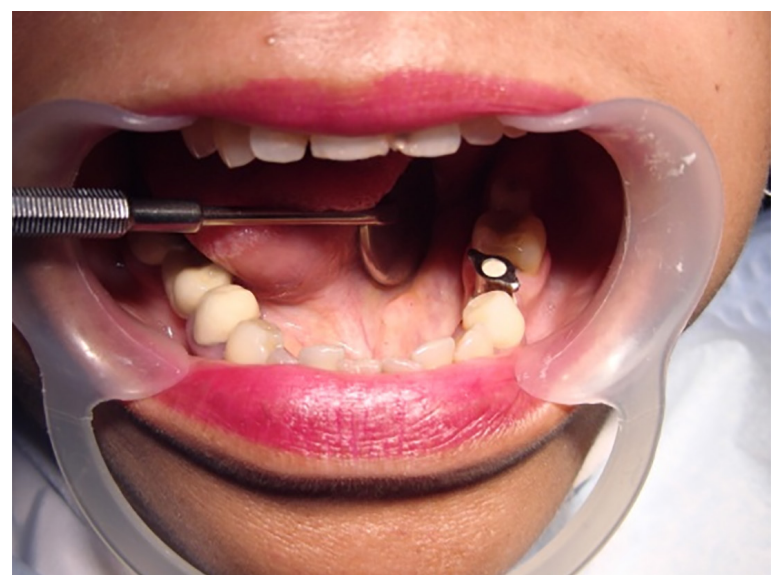

Fig. 6 Gingival cuff insertion to reform gingiva.

\section{Fourth Evaluation Visit}

A month after the third evaluation, the implant was stable in its place with no mobility found. Gingival cuff was taken off and the impression, the bite registration and the abutment cap placement were performed.

\section{Fifth Evaluation Visit}

Six weeks after fourth evaluation, the implant was stable in its place with no mobility found. Placement of temporary crown with freegenol was performed. The occlusion and articulation were good.

\section{Sixth Evaluation Visit}

Two weeks after the fifth visit and permanent insertion of the porcelain fused to metal (PFM) crown was performed (Fig. 7).

\section{Seventh Evaluation Visit}

Patient came to evaluate the treatment two weeks after the sixth visit and no complaint was recorded from the patient. Evaluation on the crown with regard to percussion test, palpation and mobility had all come out negative. Periapical radiograph was taken to examine the post insertion condition of PFM crown (Fig. 8). Patient was asked to maintain her oral hygiene dan came for evaluation in the next six months.

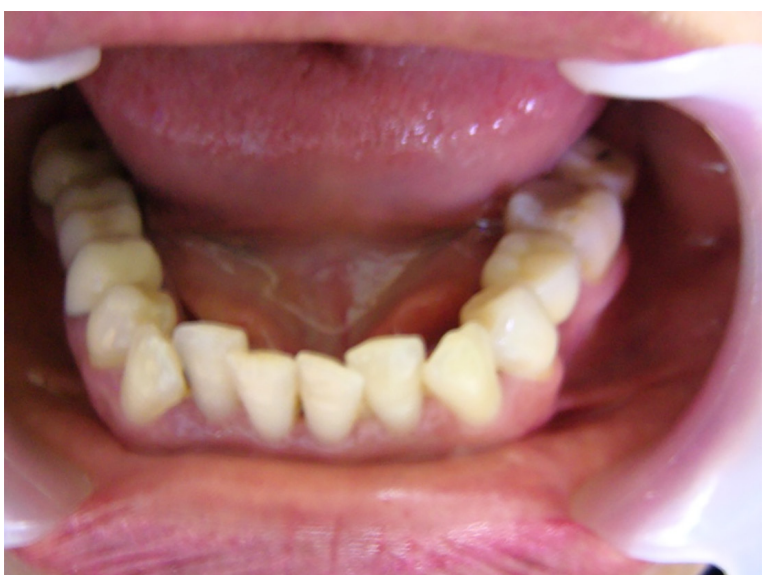

Fig. 7 Insertion of PFM crown on the mandibular left second premolar after implant placement.

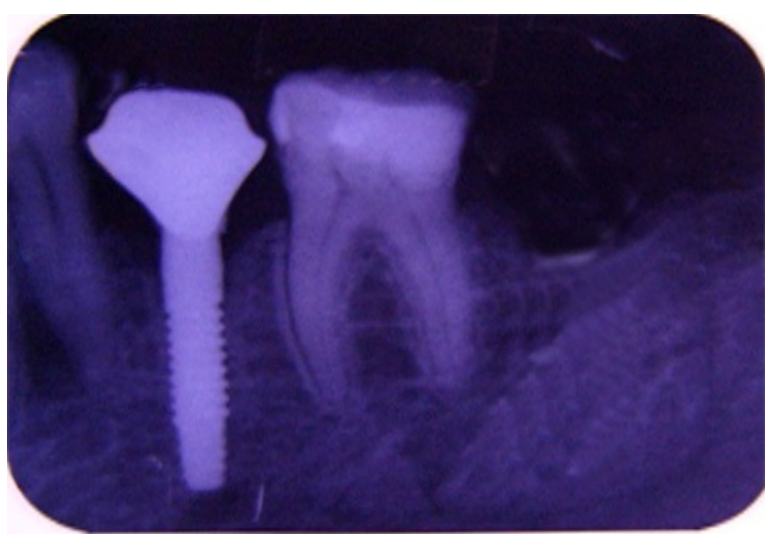

Fig. 8 Post-operative periapical radiograph with the PFM crown placement.

\section{DISCUSSION}

The goal of missing teeth rehabilitation is to obtain optimal aesthetics and function. Fixed bridge was one of the treatment options to replace one missing tooth, but it has disadvantages, such as the preparation of the abutment teeth may lead to discomfort feelings to the patient. To overcome the disadvantages of fixed bridge treatment plan, implant has been the most favourable option to replace one missing tooth. Implant provided patient with good aesthetics and convenience (Zarb et al., 2009). Implant placement procedures required surgery phase in particular method which will provide good emerging profile. Good aesthetics appearance 
of implant can be achieved by contouring gingival sulcus around it, so it will be close to natural teeth.

One-piece implant construction provides maximum strength, while its $3.0 \mathrm{~mm}$ diameter allows placement in narrow ridges with limitation in mesio-distal space. The clinically proven modified square thread form surface maximises bone-toimplant contact and provides excellent osseointegration. One-piece implant may be used for uncompromised oral health status, favourable and stable occlusal relationship, adequate mesial distal and buccal lingual bone volume, and sufficient bone density to provide initial rigid fixation (Jacobs \& O’Connell, 2011).

The tissue punch may be used to gain access to the site. This method was chosen because it provides minimum invasion flap opening. Flapless surgery will prevent the periosteum separation from soft tissue, so that the blood supply is maintained around the marginal bone, which lead to the reduction of alveolar bone resorption risk. The advantages of carrying out flapless surgery were less bleeding complication postsurgery, improvement in patient comfort, fast surgical time and acceleration of wound healing phase (Rubianto, 2018). In the present case, flapless surgery was performed because the alveolar bone morphology was adequate and did not need hard and soft tissue manipulation.

The recommended drilling sequence should be done under a constant stream of sterile irrigation, with a drill speed of $850 \mathrm{rpm}$ to $2,500 \mathrm{rpm}$. A pumping motion should be employed to help prevent overheating the bone. In the present case a bone tap was required because of the dense cortical bone. It was driven using a low-speed handpiece with $30 \mathrm{rpm}$ in a clockwise direction. Removing the bone tap was done by reversing the handpiece. One-piece implants can be used in small gaps mesiodistally to replace the missed premolars where standard diameter implants cannot be installed, therefore, could reduce the risk of damaging adjacent roots during drilling and implant placement. Additionally, more bone height is preserved between the implant and the adjacent roots, so it becomes more favourable for the long-term implant successful rate.

One-piece implant is less invasive, has simplified procedure and shortened treatment. With one-piece implant, the soft tissue experiences less trauma than two stage protocols, as it eliminates the need for second stage surgery and decreases patient exposure to additional unnecessary pain, discomfort and provides painless replacement of missing tooth. One-piece implant is usually designed with modified square-thread form with hydroxyapatite coating. It has been shown to maximise bone attachment to implant surface and intensify osseointegration to achieve high primary stability (Beumer et al., 2015). The combination of maximum strength and minimum profile would be the advantages that made one-piece implant to be considered; allowing treatment of spaces that cannot be treated with conventional twopiece implants. The titanium nitride coating on the abutment portion also improved soft tissue aesthetics.

One-piece implants should not be loaded for a minimum of three months post implantation in a high-density bone. In the less density bone, it should not be loaded for around six months after implantation (Rubianto, 2018). In the present case, as it was a highdensity bone, no loading was done onto the implant for three months. In this case, the primary stability and osseointegration were achieved easily due to the high-density bone (Hahn, 2005). Nowadays, one-piece implants are provided with different abutment types (e.g., ball type, half cylinder type) and wider ranges of implant diameter. In the present case, the one-piece implant with half cylinder type abutment ( $8 \mathrm{~mm}$ length) was used according to the available interocclusal space to the antagonist teeth. 
The one-piece implant has more simplified technique with regard to the prosthesis insertion stage. It did not require a healing abutment and the emerging profile formation is similar to the two-piece implant. Impression taking procedures for one-piece implant, can be done in three months following the implant placement. In the present case, the implant placement was successfully done. This could only be achieved by carefully designed the treatment plan and considering the following factors: the implant site location to the nerves, an adequate width and height of the alveolar bone, and the prostheses type that would be chosen.

\section{CONCLUSION}

In the missing tooth case, dental implant was a preferrable treatment plan by most patients. The benefit of one-piece implant is that the implant placement procedures are simplified, thus one-piece implant placement is considered as an alternative and acceptable treatment for the dentists and the patients.

\section{ACKNOWLEDGEMENTS}

This case report was funded by Faculty of Dentistry, Universitas Hang Tuah, Surabaya, Indonesia (Ref. no. B/1678/UHT.B0.05/ $\mathrm{VI} / 2021)$.

\section{REFERENCES}

Beumer J III, Faulkner RF, Shah KC, Moy PK (eds.) (2015). Fundamentals of Implant Dentistry. Chicago: Quintessence Publishing, pp. 13-19.

Hahn J (2005). One-piece root-form implants: A return to simplicity. F Oral Implantol, 31(2): 77-84. https://doi.org/10.1563/0 $-737.1$

Jacobs SH, O’Connell BC (2011). Dental Implant Restoration: Principles and Procedures. London: Quintessence Publishing, pp. 5569.

Misch CE (2007). Contemporary Implant Dentistry, 3rd edn. St Louis: Mosby, pp. 11-17.

Powers JM, Sakaguchi RL (2006). Craig's Restorative Dental Materials, 12th edn. St Louis: Mosby Elsevier, pp. 554-560.

Rubianto M (2018). Periodontologi 1: Kasus-kasus Pemasangan Dental Implant. Surabaya: Airlangga University Press, pp. 86-98.

Zarb GA, Bolender CL, Eckert SE (2009). Prosthodontic Treatment for Edentulous Patients: Complete Dentures and Implantsupported Prostheses, 12th edn. St Louis: Mosby, pp. 481-488. 